\title{
BMJ Open National Early Warning Score for predicting intensive care unit admission among elderly patients with influenza infections in the emergency department: an effective disposition tool during the influenza season
}

\author{
Te-Hao Wang, ${ }^{1}$ Jing-Cheng Jheng, ${ }^{1}$ Yen-Ting Tseng, ${ }^{1}$ Li-Fu Chen, ${ }^{1,2}$ \\ Jui-Yuan Chung (1) ${ }^{3,4}$
}

To cite: Wang T-H, Jheng J-C Tseng Y-T, et al. National Early Warning Score for predicting intensive care unit admission among elderly patients with influenza infections in the emergency department: an effective disposition tool during the influenza season. BMJ Open 2021;11:e044496. doi:10.1136/ bmjopen-2020-044496

- Prepublication history and additional online supplemental material for this paper are available online. To view these files, please visit the journal online (http://dx.doi.org/10. 1136/bmjopen-2020-044496).

Received 06 September 2020 Accepted 14 May 2021
Check for updates

(c) Author(s) (or their employer(s)) 2021. Re-use permitted under CC BY-NC. No commercial re-use. See rights and permissions. Published by BMJ.

For numbered affiliations see end of article.

\section{Correspondence to}

Dr Jui-Yuan Chung;

b101092138@tmu.edu.tw and

Dr Li-Fu Chen;

12206@ymuh.ym.edu.tw

\section{ABSTRACT}

Objective During the influenza epidemic season, the fragile elderlies are not only susceptible to influenza infections, but are also more likely to develop severe symptoms and syndromes. Such circumstances may pose a significant burden to the medical resources especially in the emergency department (ED). Disposition of the elderly patients with influenza infections to either the ward or intensive care unit (ICU) accurately is therefore a crucial issue.

Study design Retrospective cohort study.

Setting and participants Elderly patients ( $\geq 65$ years) with influenza visiting the ED of a medical centre between 1 January 2010 and 31 December 2015.

Primary outcome measures Demographic data, vital signs, medical history, subtype of influenza, national early warning score (NEWS) and outcomes (mortality) were analysed. We investigated the ability of NEWS to predict ICU admission via logistic regression and the receiver operating characteristic (ROC) analysis.

Results We included 409 geriatric patients in the ED with a mean age of 79.5 years and approximately equal sex ratio. The mean NEWS \pm SD was $3.4 \pm 2.9$, and NEWS $\geq 8$ was reported in $11.0 \%$ of the total patients. Logistic regression revealed that NEWS $\geq 8$ predicted ICU admission with an OR of 5.37 (95\% Cl 2.61 to 11.04). The HosmerLemeshow goodness-of-fit test was calculated as 0.95 , and the adjusted area under the ROC was 0.72. An NEWS $\geq 8$ is associated with ICU-admission and may help to triage elderly patients with influenza infections during the influenza epidemic season.

Conclusion The high specificity of NEWS $\geq 8$ to predict ICU admission in elderly patients with influenza infection during the epidemic season may avoid unnecessary ICU admissions and ensure proper medical resource allocation.

\section{INTRODUCTION}

Seasonal influenza pandemic is a global public health issue that causes approximately 5 million cases of severe complicated
Strengths and limitations of this study

- This was the first study to evaluate the use of the National Early Warning Score (NEWS) in predicting intensive care unit (ICU) admission among elderly patients with influenza infections.

- The high specificity setting of NEWS $\geq 8$ to predict ICU admission in elderly patients with influenza infection during the epidemic season, may avoid unnecessary ICU admissions and ensure proper medical resource utilisation.

- This is a single-centre retrospective cohort study.

- The subtypes of the influenza virus were not mentioned because different strains of the influenza virus may lead to different mortality rates.

influenza and 290000-650000 deaths worldwide each year. ${ }^{12}$ Of all the age groups, the elderlies are at the highest risk of developing serious complications from influenza. ${ }^{3}$ It is estimated that $90 \%$ (approximately 186000 cases) of influenza-related deaths ${ }^{4}$ and $>60 \%$ (approximately 44000 cases) of influenzarelated hospitalisations in the USA were associated with the elderlies. ${ }^{5}$ Meanwhile, up to over 30000 excess hospitalisations per year was noted in Europe, including France, Germany, Italy, UK and Ukraine, during the seasonal influenza pandemics. ${ }^{6}$ Another study reported an estimated 18000-96000 influenza-related intensive care unit (ICU) admissions in the USA annually. ${ }^{7}$

Dispositioning (discharge, admission to ward or ICU) the influenza-infected elderly patients during the influenza epidemic season is challenging because the overwhelming number of patients with influenza in hospitals may heavily burden the limited 
medical resources. Therefore, predicting ICU admission among elderly patients with influenza infections for the utilisation of medical resources appropriately is a crucial issue. There are several scoring systems in use to predict poor prognosis among elderly patients with influenza infections, for instance, the Geriatric Influenza Death (GID) score, which comprises five independent mortality predictors, including severe coma (Glasgow Coma Scale (GCS) score $\leq 8$ ), histories of cancer and coronary artery disease $(\mathrm{CAD})$, elevated $\mathrm{C}$ reactive protein (CRP) levels $(>10 \mathrm{mg} / \mathrm{dL})$ and bandaemia (>10\% band cells). Patients scoring $\geq 3$ points on this scale should be considered for ICU admission. ${ }^{8}$ However, the overcrowded emergency department (ED) during the influenza epidemic needs timely patient disposition (discharge, admission to ward or ICU), and it may take hours for the results of the laboratory variables for determination of the GID score to be revealed, which can lead to worsening the overcrowding.

The National Early Warning Score (NEWS) has the advantage of easy accessibility and prompt results because the variables of the scoring system consist of clinical vital signs, including respiration rate, oxygen saturation, systolic blood pressure, pulse rate, level of consciousness or altered mental status and body temperature. The NEWS allocates points in a weighted manner, and patients are classified into three categories according to their scores. According to Royal College of Physicians of London, patients with a low score (NEWS 1-4) should be evaluated by a competent registered nurse; those with a medium score (NEWS 5-6) should be reviewed by a wardbased doctor or an acute team nurse; and those with high scores (NEWS 27 ) should be assessed immediately by a clinical team with critical care competence and may be transferred to a higher-dependency care area. ${ }^{9}$

Although NEWS may be an effective and prompt tool for the dispositioning of patients in the influenza season, the optimal cut-off point to predict ICU admission among elderly patients with influenza infections may be different, because the physiological response to diseases may be different in the elderlies as compared with adults. Moreover, a study showed that systemic inflammatory response syndrome (SIRS) criteria $\geq 3$ among geriatric patients with influenza showed a greater accuracy for poor prognosis, which is different from the usual SIRS criteria cut-off point of 2 among non-geriatric patients. ${ }^{10}$ Therefore, we conducted this study to validate the utilisation of NEWS in predicting ICU admission among elderly patients with influenza infections.

\section{METHODS}

\section{Study design, setting and participants}

This study was conducted in the ED of Cathay General Hospital, a medical centre with 800 ward beds and 40 ED beds, located in Taipei, Taiwan. The number of patients visiting the ED was approximately 55000 annually, with $33 \%$ of elderly adult patients. ${ }^{1112}$ Elderly patients (age $\geq 65$ years) who visited the ED between
1 January 2010 and 31 December 2015, fulfilling the following criteria, were included in the study: (1) tympanic membrane (TM) temperature $\geq 37.2^{\circ} \mathrm{C}$ or an increase in baseline TM temperature $\geq 1.3^{\circ} \mathrm{C}^{1112}$; (2) influenza infections, defined as a positive influenza pharyngeal or throat swab test via de antigen detection $^{13}$; (3) antiviral therapy with either oseltamivir or zanamivir within 24 hours of the diagnosis of influenza infections. We used BD Directigen Influenza $\mathrm{A}+\mathrm{B}$ Kit for influenza test, and according to literature review, BD Directigen Influenza A \& B kit had positive predictive rate of $85.7 \%$ and negative predictive rate of $89.8 \%$ for influenza $\mathrm{A}$; positive predictive rate of $66.7 \%$ and negative predictive rate of $93.9 \%$ for influenza $\mathrm{B}^{14}$

\section{Definition of variables and primary outcomes}

There were no specific international guidelines to determine the need for ICU admission. Therefore, our critical care management committee established the ICU admission criteria as follows: (1) patients who require advanced respiratory support; (2) patients who suffer from shock or circulatory failure; (3) patients who require support for two or more of the organ systems and (4) physician's clinical judgement. The NEWS is a scoring system which comprises six simple physiological parameters including respiration rate, oxygen saturation, systolic blood pressure, pulse rate, level of consciousness or new confusion and body temperature. Each parameter was allocated with a score according to their measurement, with the magnitude of the score reflecting to what extreme the parameter varies from the baseline. Further detailed information about the NEWS is listed in online supplemental file 1 . The study endpoint was admission to the ICU.

\section{Data collection, case assignments and control groups}

A retrospective medical record review was performed on the patients who fulfilled the inclusion criteria. Demographic characteristics, patient's vital signs, influenza subtypes, laboratory data and details of past medical history, complications and mortality (table 1) were collected by an emergency physician. Required data that is not recorded in the patient's medical chart was considered negative, and the patient will be eventually excluded. The patients, who were included in the study, were categorised into the 'ICU' group and 'non-ICU' group. All variables were compared between the two groups.

\section{Statistical analyses}

The statistical priori power of this study was 0.80 calculated via G-power V.3.0, which indicate sufficient sample size to analyse this issue. Statistical analyses were performed via SPSS V.23.0 for Mac (SPSS). Continuous data that were normally distributed were presented as means \pm SDs, while continuous data that were not normally distributed were presented as 
Table 1 Characteristics of geriatric patients with influenza in the emergency department

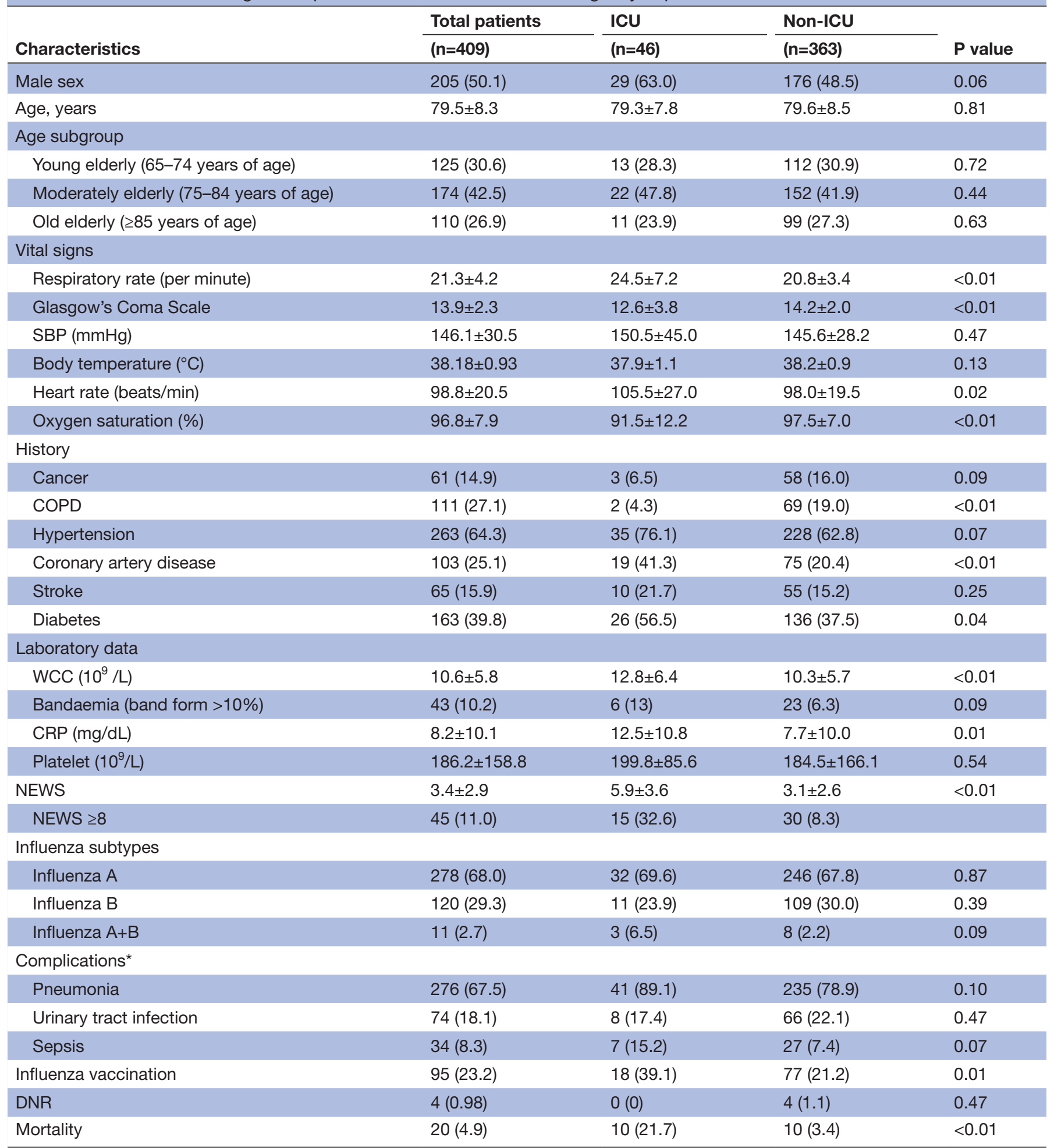

Data were presented as percentages or means \pm SD.

${ }^{*}$ Not all the complications are listed in the table.

COPD, chronic obstructive pulmonary disease; CRP, C reactive protein; DNR, do-not-resuscitate; ICU, intensive care unit; NEWS,

National Early Warning Score; SBP, systolic blood pressure; WCC, white cell count.

medians (IQRs). For independent samples, t-test or the Mann-Whitney-Wilcoxon test was used to compare continuous data. Pearson's $\chi^{2}$ test or Fisher's exact test was used for categorical variables. The area under the receiver operating characteristic curve (AUROC) was used to evaluate ICU admission discrimination ability. AUROCs were further adjusted for comorbidities that affect ICU admission. Furthermore, sensitivity, specificity, positive predictive value and negative predictive value (NPV) were also calculated. 


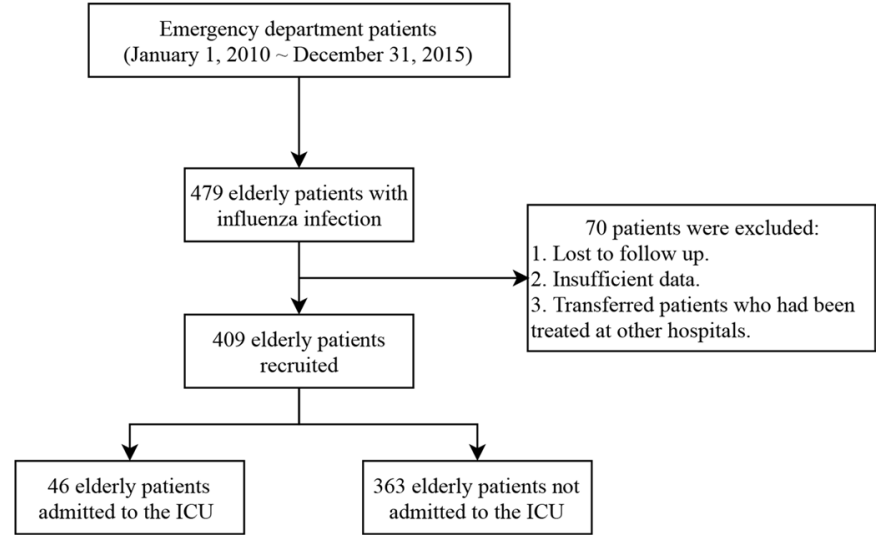

Figure 1 Flow chart of this study.

\section{Patients and public involvement}

This study did not involve patients and the public in the design or planning of the study.

\section{RESULTS}

Initially, a total of 479 geriatric patients in the ED were recruited into this study. After excluding 70 patients who were lost to follow-up, had insufficient data and transferred to be treated at other hospitals, 409 patients were eventually included (figure 1 ). The male to female ratio and the mean age $\pm \mathrm{SD}$ were approximately equal in both the groups. None of the patients signed the 'do-notresuscitate' (DNR) agreement on admission.

Vital signs showed mean $\pm \mathrm{SD}$ of the respiratory rate and heart rate were significant higher in the ICU group $(24.5 \pm 7.2 / \mathrm{min}$ and $105.5 \pm 27.0 / \mathrm{min}$, respectively) than the non-ICU group $(20.8 \pm 3.4 / \mathrm{min}$ and $98.0 \pm 19.5 / \mathrm{min}$, respectively). Furthermore, consciousness level, GCS, was lower in the ICU group at $12.6 \pm 3.8$ than the non-ICU group at $14.2 \pm 2.0$, with a $\mathrm{p}<0.01$.

For medical histories, the prevalence of $\mathrm{CAD}$ and diabetes was significantly higher in the ICU group $(41.3 \%$ and $20.4 \%)$ than in the non-ICU group $(56.5 \%$ and $37.5 \%$ ). While the laboratory data showed higher white blood cell count and CRP level in the ICU group, at $12.8 \pm 6.410^{3} / \mathrm{mm}^{3}$ and $12.5 \pm 10.8 \mathrm{mg} / \mathrm{dL}$, than the nonICU group, at $10.3 \pm 5.710^{3} / \mathrm{mm}^{3}$ and $7.7 \pm 10.0 \mathrm{mg} / \mathrm{dL}$. The mean NEWS \pm SD was significantly higher in the ICU group (5.9 \pm 3.6$)$ than in the non-ICU group (3.1 \pm 2.6$)$. The percentage of NEWS $\geq 8$ was higher in the ICU group $(32.6 \%)$ than in non-ICU group $(8.3 \%)$.

The percentages of influenza A and influenza B infections showed no significant differences between the ICU and non-ICU groups. The patients were treated with either oseltamivir or zanamivir within 24 hours of the diagnosis of influenza infections being confirmed. Complications of influenza infections included pneumonia $(67.5 \%)$, urinary tract infections $(18.1 \%)$ and sepsis $(8.3 \%)$. Influenza vaccination had shown no prominent difference between the ICU and non-ICU groups. The ICU group had a higher mortality percentage $(21.7 \%)$ than the

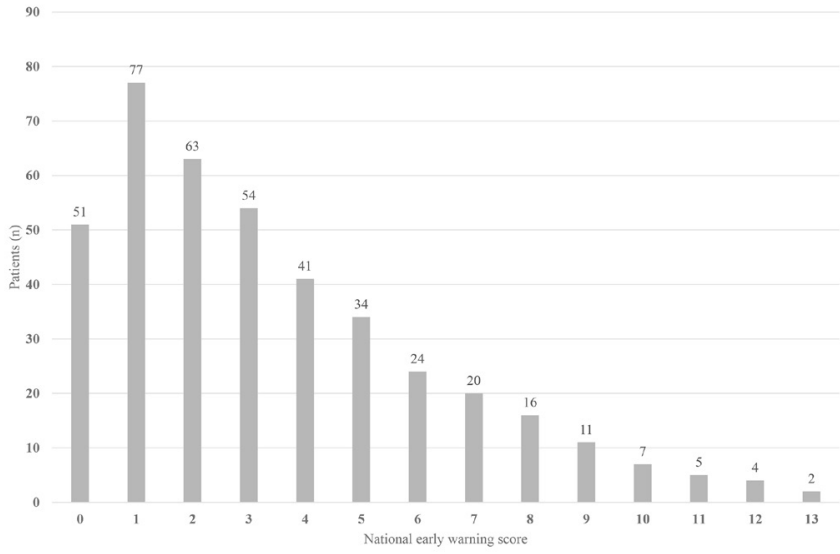

Figure 2 Distribution of patients by National Early Warning Score among elderly patients with influenza infections.

non-ICU group (3.4\%). Causes of mortality among the 20 elderly patients were sepsis (14 patients), respiratory failure (three patients), and cardiovascular events (two patients with acute myocardial infarction and one with myocarditis). No statistical difference in DNR orders was observed between the ICU and the non-ICU groups.

The distribution of elderly patients with influenza infections according to the NEWS was as follows: $51(12.5 \%)$ patients, 0 points; 77 (18.8\%), 1; 63 (15.4\%), 2; and, 218 $(53.3 \%), \geq 3$ points (figures 2 and 3 ). The Youden's index of NEWS was 0.32 , and the optimal cut-off point for NEWS to predict ICU admission among elderly patients with influenza infections was calculated as $\geq 8$ points. Univariate logistic regression showed that a NEWS $\geq 8$ predicts ICU admission with an OR of 5.37 (95\% CI 2.61 to 11.04). The Hosmer-Lemeshow goodness-of-fit score was 0.95 for NEWS $\geq 8$ (table 2).

The AUROC was adjusted for CAD $(\mathrm{p}=0.01)$ and diabetes $(\mathrm{p}=0.04)$. The adjusted AUROC for predicting ICU admission showed that NEWS $\geq 8 \quad(0.72 ; 95 \%$ CI 0.66 to 0.81 ) had an acceptable ICU admission discrimination ability (figure 4, table 3). The performance of NEWS

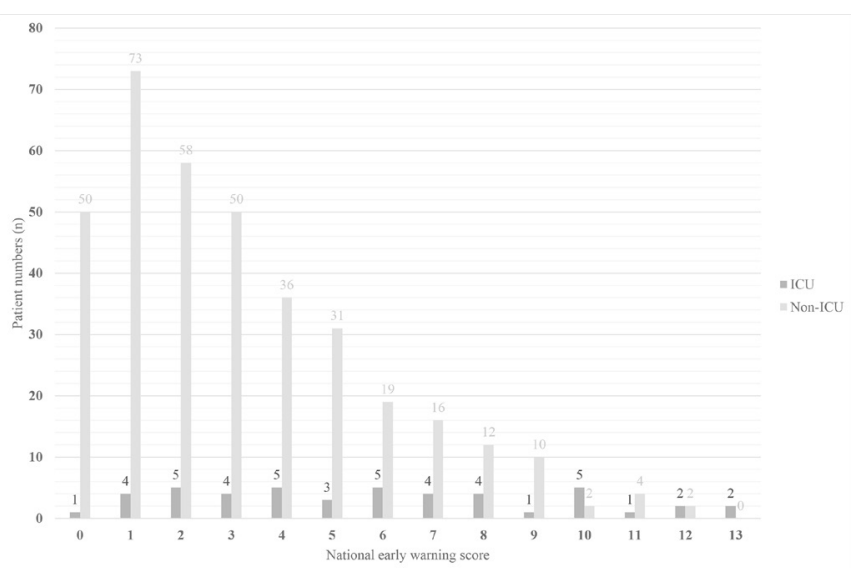

Figure 3 Distribution of patients admitted to the intensive care unit (ICU) and patients not admitted to the ICU by National Early Warning Score among elderly patients with influenza infection. 
Table 2 Predicting ICU admission among geriatric patients with influenza infections by using news score $\geq 8$, identified by univariate logistic regression

\begin{tabular}{llllll}
\hline Score & B & OR & 95\% Cl & $\begin{array}{l}\text { value } \\
\text { valu }\end{array}$ & $\begin{array}{l}\text { Hosmer-Lemeshow } \\
\text { goodness of fit }\end{array}$ \\
\hline $\begin{array}{l}\text { NEWS } \\
\geq 8\end{array}$ & 1.68 & 5.37 & $\begin{array}{l}2.61 \text { to } \\
11.04\end{array}$ & $<0.01$ & 0.95 \\
\hline
\end{tabular}

ICU, intensive care unit; NEWS, National Early Warning Score.; .

$\geq 8$ in predicting ICU admission among elderly patients with influenza infections showed a high specificity of 0.92 (95\% CI 0.88 to 0.94$)$ and a high NPV of 0.91 (95\% CI 0.90 to 0.93 ) (table 4 ).

\section{DISCUSSION}

We consider NEWS instead of NEWS2 to identify ICU admission among elderly patients with influenza infections in the ED because several studies showed NEWS2 non-superior to NEWS in discriminating patients with adverse outcomes. ${ }^{1516}$ NEWS2 was originally designed to improve safety for patients with hypercapnic respiratory failure, with oxyhemoglobin saturation by pulse oximetry $\left(\mathrm{SpO}_{2}\right)$ adjustments. However, a multicentre retrospective observational study, conducted in the UK, which identified 48898 patients, showed that NEWS2 do not improve discrimination of adverse outcomes in patients with type 2 respiratory failure, while compared with the original NEWS. ${ }^{15}$ Furthermore, a Danish study also showed similar

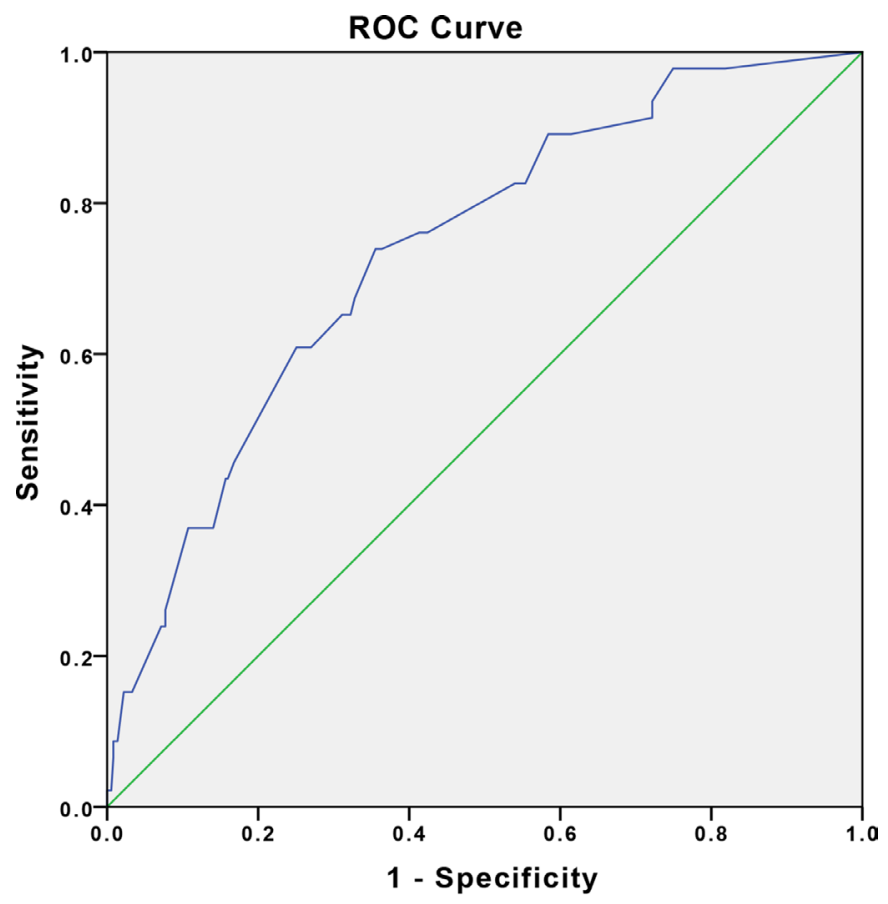

Figure 4 Area under the curve of National Early Warning Score $\geq 8$ to predict intensive care unit admission among elderly patients with influenza infection.
Table 3 Adjusted AUROC for ICU admission discrimination of NEWS score $\geq 8$ among geriatric patients with influenza infections

\begin{tabular}{llll}
\hline NEWS $\geq \mathbf{8}$ & AUROC & $\mathbf{9 5 \%}$ Cl & P value \\
\hline Adjusted & 0.72 & 0.66 to 0.81 & $<0.01$ \\
Unadjusted & 0.69 & 0.62 to 0.74 & $<0.01$ \\
\hline
\end{tabular}

AUROC, area under the receiver operating characteristic; ICU, intensive care unit; NEWS, National Early Warning Score.;

result with decreased sensitivity for 48-hour mortality and ICU admission while evaluating patients via the modified NEWS than the unmodified NEWS, and concluded that the identification of deterioration may be compromised by modifications. ${ }^{16}$

The NEWS is an early warning scoring (EWS) system developed by a multiprofessional working group by reviewing and modifying numbers of the EWS systems, especially the Vitalpac Early Warning Score (ViEWS). Adjustments were made according to clinical and professional recommendations. ${ }^{17}$ Furthermore, three points were added to every extreme value in the physiological parameters of the NEWS to overcome the paucity of extreme values in the ViEWS database. ${ }^{18}$ NEWS has also been used to identify early cardiac arrest, unanticipated ICU admission, ${ }^{19}$ acute medical admissions ${ }^{20}$ and non-elective surgical admissions ${ }^{21}$ even in prehospital settings. $^{22}$

NEWS was also capable of predicting the deterioration of patients in the ED with sepsis because Goulden et al discovered that NEWS had an equivalent or even superior value for quick sequential organ failure assessment and SIRS criteria in predicting mortality and ICU admission among patients in the ED with sepsis. ${ }^{23}$ Among patients at risk of adverse clinical outcomes, the NEWS had an equivalent performance in identifying patients similar to the Medical Emergency Team Criteria. ${ }^{24}$ A literature review conducted by Nannan Panday et al showed that ICU admission among ED patients was best predicted by NEWS. ${ }^{25}$ However, NEWS has never been validated for use in predicting ICU admission among elderly patients with influenza infections.

This study discovered that the optimal cut-off point for NEWS to predict ICU admission among influenzainfected elderly patients was 8 points, as identified by the

Table 4 Performance of NEWS score $\geq 8$ in predicting ICU admission among geriatric patients with influenza infections

$\begin{array}{ll}\text { NEWS } \geq 8 & \\ \text { Sensitivity } & 0.33(0.19-0.48) \\ \text { Specificity } & 0.92(0.88-0.94) \\ \text { Positive predictive value } & 0.33(0.23-0.46) \\ \text { Negative predictive value } & 0.91(0.90-0.93)\end{array}$

NEWS, National Early Warning Score; ICU, intensive care unit. 
Youden's index. The sensitivity, specificity, positive predictive value and NPV were $33 \%, 92 \%, 33 \%$ and $91 \%$, respectively. The high specificity of $92 \%$ and NPV of $91 \%$ may be useful in ruling out poor prognosis and ICU admission among elderly patients with influenza infection. ${ }^{8} \mathrm{~A}$ test with a high sensitivity but low specificity may result in admitting patients who are not actually severely ill to the ICU. ${ }^{26}$ In contrast, a test with high specificity and low sensitivity, similar to the performance of NEWS $\geq 8$ in the present study, may possess the advantage of a low falsepositive rate, which may avoid unnecessary ICU admissions. As medical resources are usually deficient during the influenza epidemic season, it is crucial to avoid unnecessary ICU admissions and ensure proper medical resource utilisation.

The vital signs were acquired at the triage, which could more clearly reflect the patients' primitive physiological response to the disease, because vital signs retrieved after treatments such as antipyretic therapy or fluid supplementation may affect the primitive physiological response. A possible reason for the extreme vital signs observed at the ED may be associated with the delayed seeking of medical attention by the elderly patients ${ }^{8}$ because the mean duration between onset of initial symptoms to the time of arrival in the ED was 3.56 days in this study. Patients admitted to the ICU were more likely to present with initial extreme vital signs, with a mean respiratory rate of $24.5 / \mathrm{mi}$, mean GCS score of 12.6 , mean heart rate of $105.5 / \mathrm{min}$ and a mean oxygen saturation level of $91.5 \%$. While incorporating each vital sign into the NEWS system, a sum of 8 points was calculated (individual score for each clinical vital sign: 2 points, 3 points, 1 point, and 2 points), which is compatible with our calculated cut-off of 8 points.

Influenza-infected elderly patients who were admitted to the ICU had a significantly higher percentage of a history of diabetes and CAD. A previous study showed that CAD may affect prognosis in elderly patients with influenza infections ${ }^{10}$ because influenza infections may exaggerate underlying cardiac disease and increase the chances of myocardial infarction among patients with a history of $\mathrm{CAD}{ }^{27}$ Meanwhile, patients with a history of diabetes may be more susceptible to sepsis because of the impairment of innate immune response caused by poor glycaemic control. ${ }^{28}$ The high prevalence of chronic obstructive pulmonary disease (COPD) in this study, compared with the overall geriatric population, was probable due to the impaired pulmonary function of the elderly patients with COPD, which makes them more susceptible to influenza infection. ${ }^{29}$

Although influenza vaccination plays an important role in attempts to reduce the mortality burden of influenza, the mortality benefits of influenza vaccination in elderly people is still controversial. Several studies, since 1980, were unable to proof significant decrease in influenza-related mortality, even as vaccination coverage increased from $15 \%$ to $65 \%$. In addition, the effectiveness of the influenza vaccine also varies from season to season. ${ }^{30}$ Further researches are needed to clarify this issue.

The strength of this study is that this is the first study to evaluate the use of the NEWS in predicting ICU admission among elderly patients with influenza infections. However, there were some limitations to this study. First, the severity of influenza infections may be higher because the study was conducted in a tertiary medical centre. Second, because of a retrospective chart review study setting, certain patient-related data or information may have been missed. Third, the subtypes of the influenza virus were not mentioned because different strains of the influenza virus may lead to different mortality rates. ${ }^{31}$ Fourth, the diagnosis of influenza was based on the rapid antigen test, which may have yielded false-positive or falsenegative results. Advanced examinations, such as reverse transcription PCR, immunofluorescence assay, or viral culture should be performed to confirm the diagnosis of influenza infection. However, advanced examinations are expensive and time-consuming; therefore, it is more practical to use rapid antigen test instead for its simplicity and prompt results. Fifth, this was a single-centre study, and the results of this study may not be generalisable to other countries; further external validation is needed to prove the findings of this study.

\section{CONCLUSION}

In conclusion, NEWS $\geq 8$ had the advantage of both high specificity and NPV to predict ICU admission among elderly patients with influenza infections in the ED. This suggest lower false-positive rate of ICU admission during the influenza pandemic season, and consequence in reserving medical resources for the actual critical patients.

\section{Author affiliations}

${ }^{1}$ Department of Emergency Medicine, National Yang Ming Chiao Tung University Hospital, Ilan, Taiwan

${ }^{2}$ School of Medicine, National Yang Ming Chiao Tung University, Taipei, Taiwan ${ }^{3}$ Department of Emergency Medicine, Cathay General Hospital, Taipei, Taiwan

${ }^{4}$ School of Medicine, Fu Jen Catholic University, Taipei, Taiwan

\section{Acknowledgements We thank Editage for English language editing.}

Authors contributions T-HW, J-CJ, Y-TT, L-FC and J-YC designed and conceived this study, T-HW and J-CJ wrote the manuscript. J-YC performed the statistical analysis. J-YC and L-FC provided professional suggestions. All authors read and approved the final manuscript.

Funding The authors received no specific funding for this work. The authors have not declared a specific grant for this research from any funding agency in the public, commercial or not-for-profit sectors.

Competing interests All authors denied any conflict of interest.

Patient consent for publication Not required.

Ethics approval This study was approved by the institutional Review Board of the Cathay General Hospital and was conducted according to the Declaration of Helsinki. Due to the retrospective setting of this study, the need for informed consent from the patients was waived.

Provenance and peer review Not commissioned; externally peer reviewed. Data availability statement Data available within the article or its supplementary materials 
Supplemental material This content has been supplied by the author(s). It has not been vetted by BMJ Publishing Group Limited (BMJ) and may not have been peer-reviewed. Any opinions or recommendations discussed are solely those of the author(s) and are not endorsed by BMJ. BMJ disclaims all liability and responsibility arising from any reliance placed on the content. Where the content includes any translated material, BMJ does not warrant the accuracy and reliability of the translations (including but not limited to local regulations, clinical guidelines, terminology, drug names and drug dosages), and is not responsible for any error and/or omissions arising from translation and adaptation or otherwise.

Open access This is an open access article distributed in accordance with the Creative Commons Attribution Non Commercial (CC BY-NC 4.0) license, which permits others to distribute, remix, adapt, build upon this work non-commercially, and license their derivative works on different terms, provided the original work is properly cited, appropriate credit is given, any changes made indicated, and the use is non-commercial. See: http://creativecommons.org/licenses/by-nc/4.0/.

ORCID iD

Jui-Yuan Chung http://orcid.org/0000-0002-4823-555X

\section{REFERENCES}

1 World Health organization. Available: https://www.who.int/newsroom/detail/14-12-2017-up-to-650-000-people-die-of-respiratorydiseases-linked-to-seasonal-flu-each-year [Accessed 25 Aug 2020].

2 Dawood FS, Iuliano AD, Reed C, et al. Estimated global mortality associated with the first 12 months of 2009 pandemic influenza A H1N1 virus circulation: a modelling study. Lancet Infect Dis 2012;12:687-95.

3 Mertz D, Kim TH, Johnstone J, et al. Populations at risk for severe or complicated avian influenza H5N1: a systematic review and metaanalysis. PLoS One 2014;9:e89697.

4 Thompson WW, Shay DK, Weintraub E, et al. Influenza-Associated hospitalizations in the United States. JAMA 2004;292:1333-40.

5 Thompson WW, Shay DK, Weintraub E, et al. Mortality associated with influenza and respiratory syncytial virus in the United States. JAMA 2003;289:179-86.

6 Gasparini R, Amicizia D, Lai PL, et al. Clinical and socioeconomic impact of seasonal and pandemic influenza in adults and the elderly. Hum Vaccin Immunother 2012;8:21-8.

7 Chow EJ, Doyle JD, Uyeki TM. Influenza virus-related critical illness: prevention, diagnosis, treatment. Crit Care 2019;23:214.

8 Chung J-Y, Hsu C-C, Chen J-H, et al. Geriatric influenza death (GID) score: a new tool for predicting mortality in older people with influenza in the emergency department. Sci Rep 2018;8:9312.

9 Hawkes N. Royal College recommends national system to recognise deteriorating patients. BMJ 2012;345:e5041.

10 Tai HC-H, Yeh C-C, Chen Y-A, et al. Utilization of systemic inflammatory response syndrome criteria in predicting mortality among geriatric patients with influenza in the emergency department. BMC Infect Dis 2019;19:639.

11 Huang C-C, Kuo S-C, Chien T-W, et al. Predicting the hyperglycemic crisis death (PHD) score: a new decision rule for emergency and critical care. Am J Emerg Med 2013;31:830-4.

12 Chung M-H, Huang C-C, Vong S-C, et al. Geriatric fever score: a new decision rule for geriatric care. PLoS One 2014;9:e110927.

13 Chung $\mathrm{M}-\mathrm{H}$, Chu F-Y, Yang T-M, et al. Hypotension, bedridden, leukocytosis, thrombocytopenia and elevated serum creatinine predict mortality in geriatric patients with fever. Geriatr Gerontol Int 2015;15:834-9.

14 Gröndahl B, Puppe W, Weigl J, et al. Comparison of the BD Directigen flu A+B kit and the Abbott TestPack RSV with a multiplex RT-PCR ELISA for rapid detection of influenza viruses and respiratory syncytial virus. Clin Microbiol Infect 2005;11:848-50.

15 Pimentel MAF, Redfern OC, Gerry S, et al. A comparison of the ability of the National early warning score and the National early warning score 2 to identify patients at risk of in-hospital mortality: a multicentre database study. Resuscitation 2019;134:147-56.

16 Pedersen NE, Rasmussen LS, Petersen JA, et al. Modifications of the National early warning score for patients with chronic respiratory disease. Acta Anaesthesiol Scand 2018;62:242-52.

17 Prytherch DR, Smith GB, Schmidt PE, et al. ViEWS--Towards a national early warning score for detecting adult inpatient deterioration. Resuscitation 2010;81:932-7.

18 Royal College of physicians. Available: https://www.rcplondon.ac.uk/ file/32/download?token=vfwDKQVS [Accessed 25 Aug 2020].

19 Smith GB, Prytherch DR, Meredith P, et al. The ability of the National early warning score (news) to discriminate patients at risk of early cardiac arrest, unanticipated intensive care unit admission, and death. Resuscitation 2013;84:465-70.

20 Kovacs C, Jarvis SW, Prytherch DR, et al. Comparison of the National early warning score in non-elective medical and surgical patients. Br J Surg 2016;103:1385-93.

21 Silcock DJ, Corfield AR, Gowens PA, et al. Validation of the National early warning score in the prehospital setting. Resuscitation 2015;89:31-5.

22 Shaw J, Fothergill RT, Clark S, et al. Can the prehospital national early warning score identify patients most at risk from subsequent deterioration? Emerg Med J 2017;34:533-7.

23 Goulden R, Hoyle M-C, Monis J, et al. qSOFA, SIRS and news for predicting inhospital mortality and ICU admission in emergency admissions treated as sepsis. Emerg Med J 2018;35:345-9.

24 Smith GB, Prytherch DR, Jarvis S. A comparison of the ability of the physiologic components of medical emergency team criteria and the U. K. National Early Warning Score to discriminate patients at risk of a range of adverse clinical outcomes. Crit Care Med 2016;44:2171-81.

25 Nannan Panday RS, Minderhoud TC, Alam N, et al. Prognostic value of early warning scores in the emergency department (ED) and acute medical unit (AMU): a narrative review. Eur J Intern Med 2017;45:20-31

26 Ranganathan P, Aggarwal R. Common pitfalls in statistical analysis: Understanding the properties of diagnostic tests - Part 1. Perspect Clin Res 2018;9:40-3.

27 Mamas MA, Fraser D, Neyses L. Cardiovascular manifestations associated with influenza virus infection. Int $J$ Cardiol 2008;130:304-9.

28 Trevelin SC, Carlos D, Beretta M, et al. Diabetes mellitus and sepsis: a challenging association. Shock 2017;47:276-87.

29 Wang Y-C, Lin J-M, Li C-Y, et al. Prevalence and risks of chronic airway obstruction: a population cohort study in Taiwan. Chest 2007;131:705-10.

30 Simonsen L, Taylor RJ, Viboud C, et al. Mortality benefits of influenza vaccination in elderly people: an ongoing controversy. Lancet Infect Dis 2007;7:658-66.

31 Arbeitskreis Blut B. Untergruppe «Bewertung Blutassoziierter Krankheitserreger». Influenza Virus. Transfus Med Hemother 2009;36:32-9. 